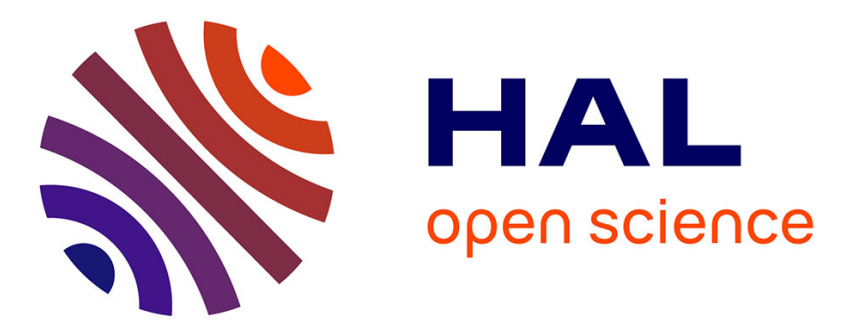

\title{
The Effect of Injection Current and Temperature on Signal Strength in a Laser Diode Optical Feedback Interferometer
}

Jalal Al Roumy, Julien Perchoux, Yah Leng Lim, Thomas Taimre, Aleksandar D. Rakic, Thierry Bosch

\section{To cite this version:}

Jalal Al Roumy, Julien Perchoux, Yah Leng Lim, Thomas Taimre, Aleksandar D. Rakic, et al.. The Effect of Injection Current and Temperature on Signal Strength in a Laser Diode Optical Feedback Interferometer. Applied optics, 2015, 54 (2), pp.312-318. 10.1364/AO.54.000312 . hal-01187185

\author{
HAL Id: hal-01187185 \\ https://hal.science/hal-01187185
}

Submitted on 26 Aug 2015

HAL is a multi-disciplinary open access archive for the deposit and dissemination of scientific research documents, whether they are published or not. The documents may come from teaching and research institutions in France or abroad, or from public or private research centers.
L'archive ouverte pluridisciplinaire HAL, est destinée au dépôt et à la diffusion de documents scientifiques de niveau recherche, publiés ou non, émanant des établissements d'enseignement et de recherche français ou étrangers, des laboratoires publics ou privés.

\section{(1)(1) $\$(0)$}

Distributed under a Creative Commons Attribution - NonCommercial - ShareAlikel 4.0 


\title{
The Effect of Injection Current and Temperature on Signal Strength in a Laser Diode Optical Feedback Interferometer
}

\author{
Jalal Al Roumy, ${ }^{1,2}$ Julien Perchoux, ${ }^{1,2, *}$ Yah Leng Lim, ${ }^{3}$ \\ Thomas Taimre, ${ }^{4}$ Aleksandar D. Rakić, ${ }^{3}$ and Thierry Bosch ${ }^{1,2}$ \\ ${ }^{1}$ CNRS, LAAS, 7 Avenue du Colonel Roche, F-31077 Toulouse Cedex 4, France \\ ${ }^{2}$ Univ de Toulouse, INP, LAAS, F-31400 Toulouse, France \\ ${ }^{3}$ School of Information Technology and Electrical Engineering, \\ The University of Queensland, QLD 4072, Australia \\ ${ }^{4}$ School of Mathematics and Physics, The University of Queensland, QLD 4072, Australia
}

compiled: November 27, 2014

\begin{abstract}
We present a simple analytical model that describes the injection current and temperature dependence of optical feedback interferometry signal strength for a single-mode laser diode. The model is derived from the Lang and Kobayashi rate equations, and is developed both for signals acquired from the monitoring photodiode (proportional to the variations in optical power) and for those obtained by amplification of the corresponding variations in laser voltage. The model shows that both the photodiode and the voltage signal strengths are dependent on the laser slope efficiency, which itself is a function of the injection current and the temperature. Moreover, the model predicts that photodiode and voltage signal strengths depend differently on injection current and temperature. This important model prediction was proven experimentally for a near-infrared distributed feedback laser by measuring both types of signals over a wide range of injection currents and temperatures. Therefore, this simple model provides important insight into the radically different biasing strategies required to achieve optimal sensor sensitivity for both interferometric signal acquisition schemes.
\end{abstract}

\section{Introduction}

Optical feedback interferometry (OFI) is a promising sensing technique for both industrial and laboratory environments due to its simple optical setup relative to other interferometric techniques. Typical sensing applications of OFI are the measurement of displacement, distance, and velocity [1]. OFI signals can be acquired by two different means: (i) by observing power fluctuations in the light emitted by the rear facet of the laser diode with a monitoring photodiode (denoted here as the PD signal), or (ii) by amplifying voltage variations across the laser terminals (denoted here as the LV signal) [2]. The latter is the only viable measurement approach when a monitoring photodiode is not included in the laser diode package, for example when an array of laser diodes is used [3]. For both methods of signal acquisition, in order to achieve the best performance of the OFI sensor it is essential to maintain the signal-to-noise ratio (SNR) at a maximum.

The dependence of OFI signal strength on bias current has been investigated previously in experiment [4-6], with earlier models providing important insight into the genesis of the interferometric signal $[2,7,8]$. Experimental results reported to date suggest that the relationship between OFI signal strength and bias current depends

\footnotetext{
* Corresponding author: julien.perchoux@laas.fr
}

on both the laser structure [5,6,9-11] and the level of feedback applied $[12,13]$. In this paper we propose a simple model, applicable to single-mode laser diodes, which provides compact analytical expressions to quantitatively describe this phenomenon of practical significance. Due to the device-dependent nature of optical feedback models, we have limited our study to singlemode (transverse and longitudinal) laser diodes. Our simple analytical model is based on that of Lang and Kobayashi [14], and describes the dependence of OFI signal strength on injection current and temperature for a single-mode laser diode. Our proposed model provides compact analytic expressions which quantitatively describe the practically significant difference in injection current and temperature dependence of interferometric $\mathrm{PD}$ and LV signals.

\section{Theoretical Model}

The rate equations for such a laser subjected to optical feedback from a distant target were first proposed by Lang and Kobayashi [14]. Our analysis begins with the version of these rate equations proposed by Donati [8]:

$$
\frac{\mathrm{d} E}{\mathrm{~d} t}=\frac{1}{2}\left[G_{N}\left(N-N_{\mathrm{tr}}\right)-\frac{1}{\tau_{p}}\right] E+\kappa E\left(t-\tau_{D}\right) \cos \left(\omega \tau_{D}\right),
$$

$$
\frac{\mathrm{d} N}{\mathrm{~d} t}=\frac{I}{q V}-\frac{N}{\tau_{e}}-G_{N}\left(N-N_{\mathrm{tr}}\right) E^{2} .
$$


In these equations, $E$ is the amplitude of the electric field, $G_{N}=v_{\mathrm{gr}} \partial g / \partial N$ is the stimulated emission gain where $v_{\text {gr }}$ is the group velocity and $g$ is the gain coefficient, $N$ is the carrier density, $N_{\text {tr }}$ is the carrier density at transparency, $\tau_{p}$ is the photon lifetime within the laser cavity, $\tau_{D}$ is the external round-trip time of flight, $\omega$ is the angular frequency of the optical field, $I$ is the current injected into the active region of the device, $q$ is the electron charge, $V$ is the volume of the active region, $\tau_{e}$ is the carrier lifetime, and $\kappa$ the cavity coupling coefficient defined as [14]:

$$
\kappa=\frac{1}{\tau_{L}}\left(1-R_{2}\right) \sqrt{\frac{R_{\mathrm{ext}}}{R_{2}}},
$$

with $\tau_{L}$ the laser cavity round-trip time, $R_{2}$ the reflectivity of the laser cavity's emitting mirror, and $R_{\text {ext }}$ the effective reflectivity of the distant target (the ratio of back-scattered power successfully coupled into the cavity to the emitted power).

Solving (1) and (2) in temporal steady state when $\kappa \tau_{p} \tau_{L} \ll \tau_{D} / 2$ and making use of $S \propto|E|^{2}$ where $S$ is the photon density, leads to the following form for the emitted power $P$ under optical feedback as a function of the power emitted by the solitary laser diode $P_{0}$ :

$$
P=P_{0}\left[1+m \cos \left(\omega \tau_{D}\right)\right],
$$

with modulation index $m$ which can be expressed as [15]:

$$
m=2 \kappa \tau_{p}\left(1+\frac{1}{S_{0} G_{N} \tau_{e}}\right),
$$

where $S_{0}$ is the steady-state value for the photon density.

The change in optical power $P$ is usually detected via a photodiode integrated within the laser package. It is the variation in photo-current obtained from this photodiode which we refer to as the OFI PD signal. Under moderate or strong feedback, the relation (4) produces sawtooth-like signals [16]. In these cases, where high back-scattered power couples into the laser cavity, the signal amplitude is strongly affected by the variation in the laser frequency $\omega$ due to feedback. For the remainder of this article, we consider only the weak feedback regime $\left(C \equiv \kappa \tau_{D} \sqrt{1+\alpha^{2}} \leq 1\right.$ where $\alpha$ is the linewidth enhancement factor and $C$ is Acket's characteristic feedback parameter $[15,17])$ in which the signal shape is sinusoidal.

With this in mind, from the rate equations solved in the steady state regime we can obtain:

$$
\frac{1}{\tau_{p}}=G_{N}\left(N_{\mathrm{th}}-N_{\mathrm{tr}}\right)
$$

where $N_{\text {th }}$ is the carrier density at threshold. Using (6) with (5) and expressing the steady-state photon density $S_{0}$ as a function of the injection current, we obtain:

$$
m=2 \kappa \tau_{p}\left(1+\frac{I_{\mathrm{th}}\left(N_{\mathrm{th}}-N_{\mathrm{tr}}\right)}{N_{\mathrm{th}}\left(I-I_{\mathrm{th}}\right)}\right),
$$

where $I_{\mathrm{th}}$ is the threshold current.

From (4), the amplitude of the power variations is $\Delta P=m P_{0}$ and, on substituting $P_{0}=\eta(I)\left(I-I_{\mathrm{th}}\right)$ where $\eta(I)$ is the laser diode slope efficiency (which is constant for an ideal laser diode), we obtain:

$$
\Delta P=2 \kappa \tau_{p} \eta(I)\left(I-I_{\mathrm{th}} \frac{N_{\mathrm{tr}}}{N_{\mathrm{th}}}\right) .
$$

The expression (8) explicitly shows how the interferometric PD signal changes with respect to the parameters of the OFI system, and in particular with injected current $I$. For an ideal laser diode, whose slope efficiency is independent of injected current, the amplitude of the PD signal is therefore a linearly increasing function of the injection current. Thus, for an ideal laser diode, the maximum interferometric signal amplitude will be attained for high injection current.

The change in carrier density $\Delta N$ leads to the corresponding change in voltage across the laser junction. It is this variation across the laser junction which we refer to as the OFI LV signal. The relationship between the OFI LV signal and the change in carrier density causing it can be obtained through the linearised relation between carrier density and junction voltage [18]:

$$
\Delta v=\frac{2 k T}{q N_{\mathrm{th}}} \Delta N
$$

where $k$ is Boltzmann's constant and $T$ is the laser temperature. Solving (2) in the small signal approximation yields:

$$
\Delta N=\frac{G_{N}\left(N_{\mathrm{th}}-N_{\mathrm{tr}}\right)}{\frac{1}{\tau_{e}}+G_{N} S_{0}} \Delta S,
$$

which can be simplified using (6) to become:

$$
\Delta N=\frac{\tau_{e}}{\tau_{p}} \cdot \frac{\Delta S}{1+G_{N} S_{0} \tau_{e}} .
$$

By combining (5) and (11), we obtain:

$$
\Delta N=\frac{\tau_{e}}{\tau_{p}}\left(m-2 \kappa \tau_{p}\right) S_{0} .
$$

By eliminating $m$ using (7) we obtain:

$$
\Delta N=2 \kappa \tau_{e} S_{0} \frac{I_{\mathrm{th}}\left(N_{\mathrm{th}}-N_{\mathrm{tr}}\right)}{N_{\mathrm{th}}\left(I-I_{\mathrm{th}}\right)} .
$$

Making use of the relationship between the steady-state photon density $S_{0}$ and the injection current:

$$
S_{0}=\frac{\tau_{p}}{V \hbar \omega} \eta(I)\left(I-I_{\mathrm{th}}\right)
$$

where $\hbar$ is the reduced Planck's constant, we arrive at the expression for the change in LV signal $\Delta v$ :

$$
\Delta v=4 \kappa \tau_{e} \eta(I) \frac{\tau_{p}}{V \hbar \omega} \cdot \frac{k T}{q N_{\mathrm{th}}} I_{\mathrm{th}}\left(1-\frac{N_{\mathrm{tr}}}{N_{\mathrm{th}}}\right) .
$$


Finally, solving the carrier rate equation (2) at threshold in the steady-state regime allows (15) to be expressed in a more compact form:

$$
\Delta v=4 \kappa \tau_{p} \eta(I) \frac{k T}{\hbar \omega}\left(1-\frac{N_{\mathrm{tr}}}{N_{\mathrm{th}}}\right) .
$$

Equation (16), which explicitly shows how the interferometric LV signal changes with respect to the parameters of the OFI system, has very interesting consequences. For an ideal laser diode, the amplitude of the LV signal does not depend on the injected current - quite unlike the amplitude of the PD signal in (8) [2]. Moreover, (16) suggests that the amplitude of the LV signal increases with temperature - a phenomenon which has been observed in experiment $[3,6]$. However, constant slope efficiency is often unrealistic in practice, especially for single-mode laser diodes where thermal effects have significant impact on the non-radiative recombination and spontaneous emission rates [19]. Indeed, slope efficiency is usually observed to decrease with injection current, leading to a corresponding decrease in the amplitude of the LV signal. Hence, in practice, the maximum strength of the LV signal is usually attained at low injection currents, just above the laser threshold $[6,20]$.

Therefore, to account for the effects of temperature on laser threshold current and slope efficiency in (8) and (16) for single-mode in-plane laser diodes, namely that an increase in temperature induces an increase of the threshold current and a decrease of the slope efficiency, we model both relationships through the use of simple exponential functions [19]:

$$
I_{\mathrm{th}}(T+\Delta T)=I_{\mathrm{th}}(T) \mathrm{e}^{\frac{\Delta T}{T_{0}}},
$$

and

$$
\eta(T+\Delta T)=\eta(T) \mathrm{e}^{-\frac{\Delta T}{T_{\eta}}},
$$

where $T_{0}$ and $T_{\eta}$ are the characteristic temperatures of the laser diode which determine the sensitivity of the laser diode to temperature changes.

\section{Experimental Setup}

In order to experimentally validate the injection current and temperature dependence of both PD and LV signal strengths in the proposed model, we have performed a series of signal amplitude measurements at different bias currents and temperatures using a simple Doppler velocimeter described in Fig. 1.

The laser diode used was a DFB laser (ML725B11F, $\lambda=1310 \mathrm{~nm}$ ) with a monitoring photodiode, integrated in a $5.6 \mathrm{~mm}$ TO-can package. A single lens (C240TME$\mathrm{C}$, focal length $f=8 \mathrm{~mm}$, numerical aperture $\mathrm{NA}=$ 0.5 , anti-reflective coating $1050-1620 \mathrm{~nm}$ ) was used to focus the laser beam onto the target, which was a rotating aluminium disk with sandblasted surface. A neutral density filter was introduced into the optical path

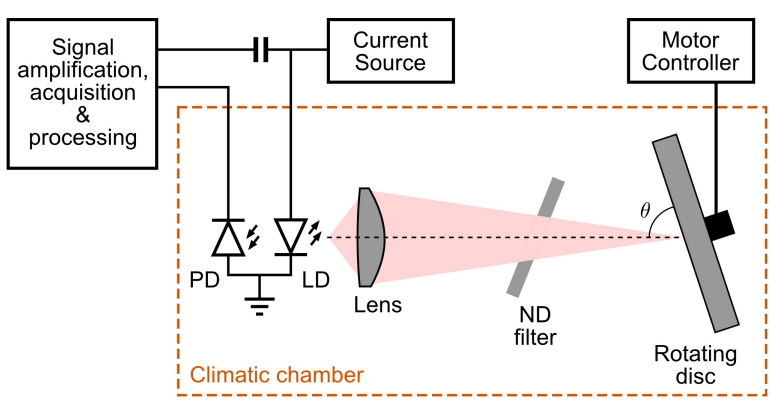

Fig. 1. Experimental setup for measuring velocity of a rotating disk. The disk is tilted around the vertical axis by $10^{\circ}$ to produce a small velocity component in the direction of the laser beam.

between the lens and the target to control the amount of optical feedback. This was to ensure that the optical feedback remained weak as the injection current or temperature was varied. The complete opto-mechanical system was placed in a climatic chamber (model: EXCAL 2221-TA) which provides temperature control over the range $-40{ }^{\circ} \mathrm{C}$ to $80^{\circ} \mathrm{C}$. The laser was driven from a current source (custom built laser driver) controlled by a Labview program through a National Instruments data acquisition card (model: NI-6251). Both PD and LV signals were amplified and acquired concurrently using the same card at the sampling rate of $1 \mathrm{MS} / \mathrm{s}$. Fast Fourier transforms were performed on the acquired time-domain signals, thereby giving the spectra of the OFI signals. The velocity of the disk was maintained constant to give a central Doppler frequency close to $50 \mathrm{kHz}$ at every temperature. The OFI signal strengths at the central Doppler frequency were then recorded.

\section{Results and discussion}

Figure 2(a) shows light-current curves measured for temperatures ranging from $-40{ }^{\circ} \mathrm{C}$ to $80{ }^{\circ} \mathrm{C}$ in steps of $5{ }^{\circ} \mathrm{C}$ using the monitoring photodiode with a dedicated low-noise transimpedance amplifier $(60 \mathrm{dBV} / \mathrm{A})$. Solid lines in Fig. 2(b) show the corresponding threshold current (calculated using the second derivative method) and the maximum slope efficiency (obtained from the first derivative of the light-current curve) [21]. Over this wide range of temperatures, an increase of the threshold current from $2.3 \mathrm{~mA}$ to $18.3 \mathrm{~mA}$ is observed together with a decrease from $0.66 \mathrm{~W} / \mathrm{A}$ to $0.38 \mathrm{~W} / \mathrm{A}$ of the maximum slope efficiency. Broken lines superimposed on Fig. 2(b) are fitted curves of the form (17) and (18), resulting in characteristic temperatures $T_{0}=42{ }^{\circ} \mathrm{C}$ and $T_{\eta}=120^{\circ} \mathrm{C}$, which show good agreement with experiment. To account for the non-ideality of the laser diode's slope efficiency, at each temperature we fitted a thirdorder polynomial to the measured light-current curve in the least-squares sense. This provides a simple behavioural model for the slope efficiency in terms of current.

At every temperature step, the behavioural model was 
(a)

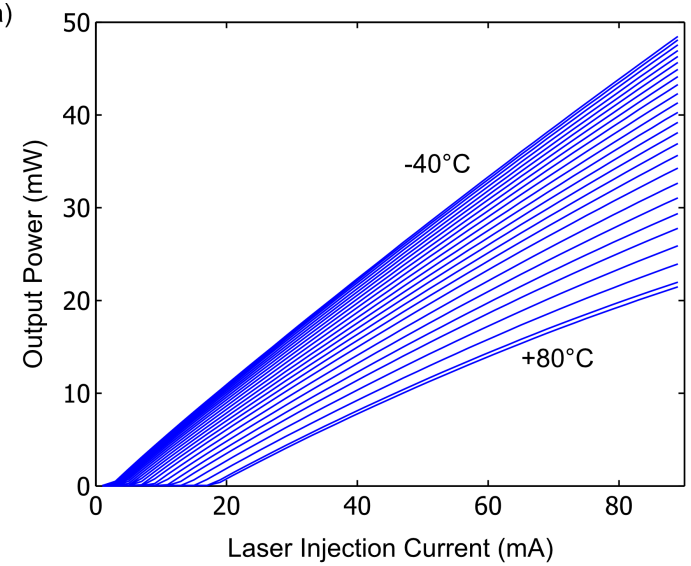

(b)

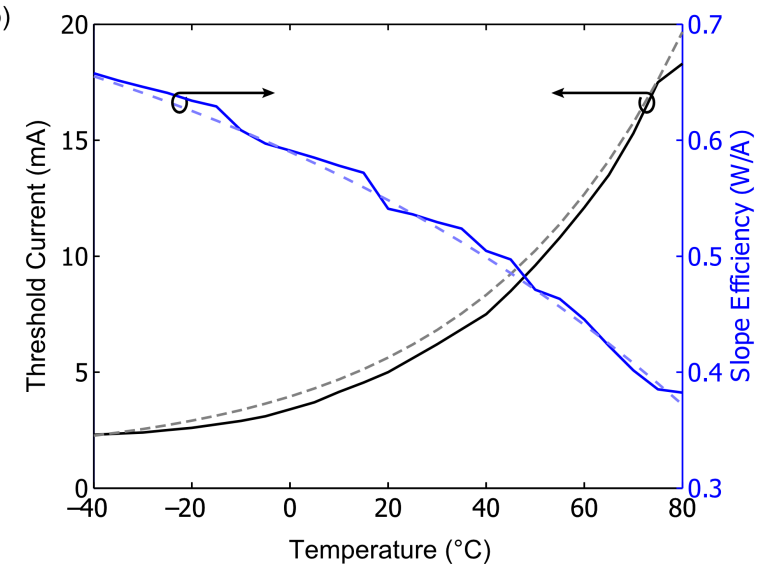

Fig. 2. (a) Output power as a function of laser injection current at different temperatures (in the temperature range from $-40{ }^{\circ} \mathrm{C}$ to $+80{ }^{\circ} \mathrm{C}$ in steps of $5{ }^{\circ} \mathrm{C}$ ), and (b) slope efficiency and threshold current as a function of temperature. Slope efficiency decreases with temperature while injection current threshold increases.

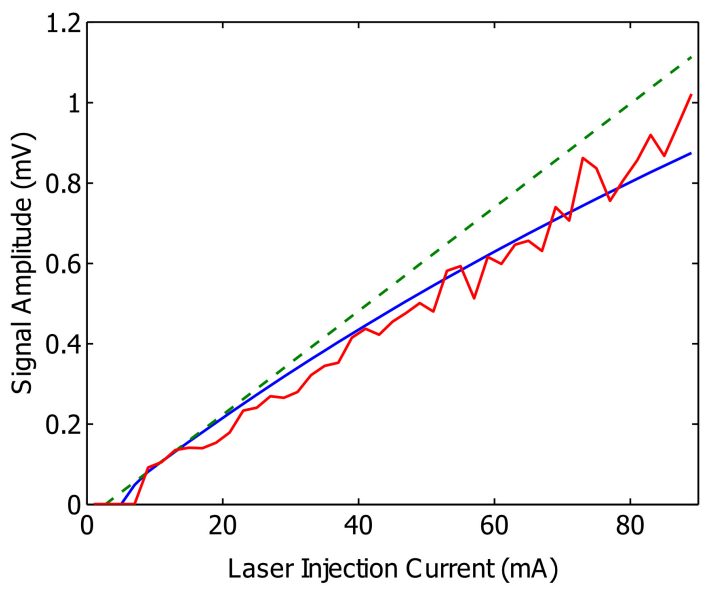

Fig. 3. Dependence of the PD signal strength on injection current: measured (red solid); modelled (constant slope efficiency of an ideal laser diode, green dashed); modelled (behavioural slope efficiency, blue solid).

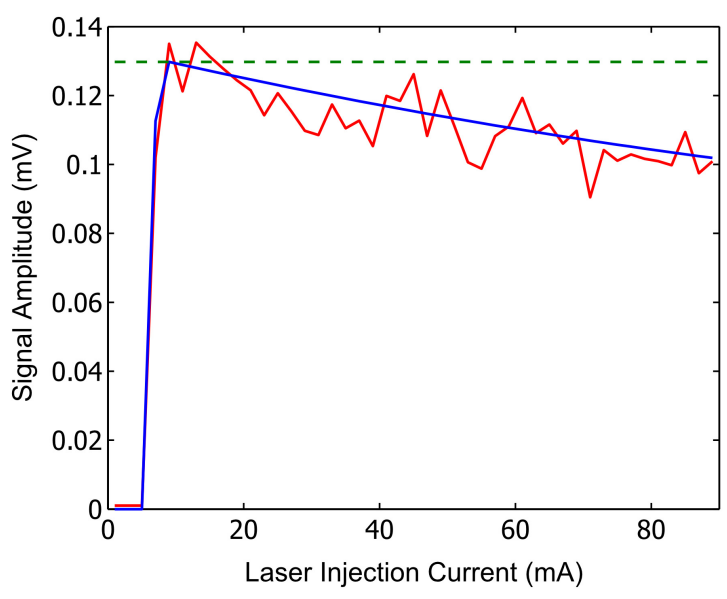

Fig. 4. Dependence of the LV signal strength on injection current: measured (red solid); modelled (constant slope efficiency of an ideal laser diode, green dashed); modelled (behavioural slope efficiency, blue solid).

determined by associating the measured threshold current and slope efficiency to the intrinsic laser parameters given in [19], and accounting for the experimental level of the cavity coupling coefficient $\kappa$ and subsequent signal amplification by including a fixed scaling factor. Figure 3 shows the measured OFI PD signal at $T=25{ }^{\circ} \mathrm{C}$ (red solid line, caused by the fluctuations in optical power) and two model curves: (i) constant slope efficiency (green broken line, ideal laser diode), and (ii) behavioural slope efficiency (blue solid line, actual laser diode). By taking into account the actual slope efficiency, the model shows good agreement with measurement over a wide range of injection current levels.

Figure 4 shows the measured LV signal at $T=25{ }^{\circ} \mathrm{C}$ (red solid line) and two model curves: (i) constant slope efficiency (green broken line), and (ii) behavioural slope efficiency (blue solid line). Figure 4 shows that the LV signal has a prominent peak slightly above the laser threshold and monotonically decreases after that. This decrease in LV signal with injection current is well explained by the corresponding decrease in slope efficiency.

The effect of temperature on the PD and LV signal strengths at a laser injection current of $59 \mathrm{~mA}$ (up to 25 times the threshold current over the entire temperature range) is shown in Fig. 5 and Fig. 6, respectively. The model using behavioural slope efficiency (blue solid line) agrees well with the experimentally-observed decrease in signal strength at high temperatures (red solid line), highlighting the importance of the temperaturedependent slope efficiency.

These results show that an increase in either injection current or temperature results in a decrease in slope efficiency. Therefore, it is important to capture the simultaneous impact of injection current and temperature on OFI signal strength. Figures 7 and 8 show the measured and modelled PD and LV signals, respectively, as a function of both the laser injection current and temper- 


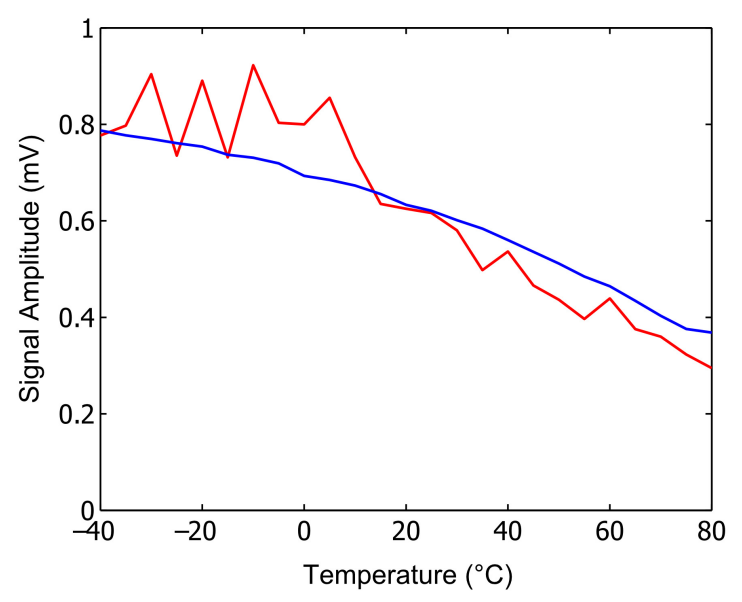

Fig. 5. Dependence of the PD signal strength on temperature: measured (red solid); modelled (behavioural slope efficiency, blue solid).

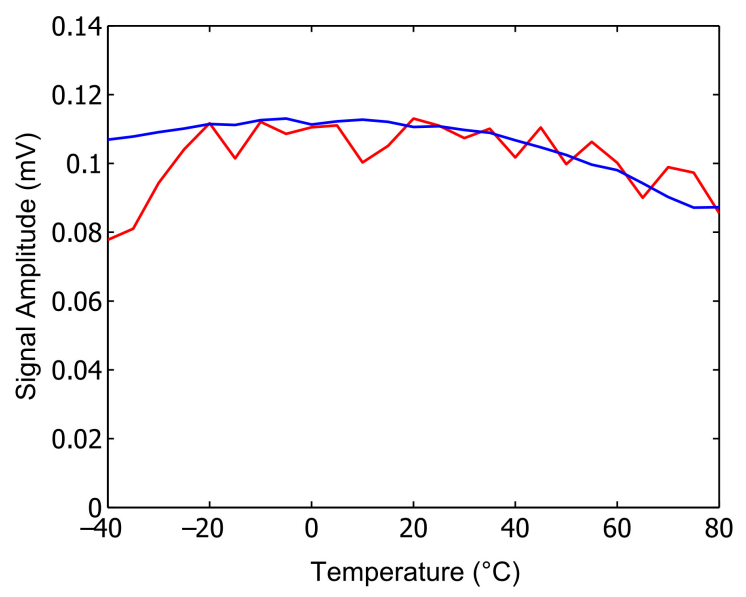

Fig. 6. Dependence of the LV signal strength on temperature: measured (red solid); modelled (behavioural slope efficiency, blue solid).

ature. The PD signal, modelled with behavioural slope efficiency [Fig. 7(b)], shows good agreement with the measured signal [Fig. 7(a)] over the entire range of injection currents and temperatures. The influence of the slope efficiency on the PD signal strength can be directly observed through the decrease of the signal strength at high temperatures.

Similarly, the LV signal, simulated with behavioural slope efficiency [Fig. 8(b)], shows good agreement with the measured signal [Fig. 8(a)]. LV signal peaks for mid-range temperatures; low temperature range is dominated by direct relationship between voltage and temperature through Eq. (9); at higher temperatures, the decrease in slope efficiency becomes the dominant factor, and the net result is a sharp decrease in signal strength. (a)

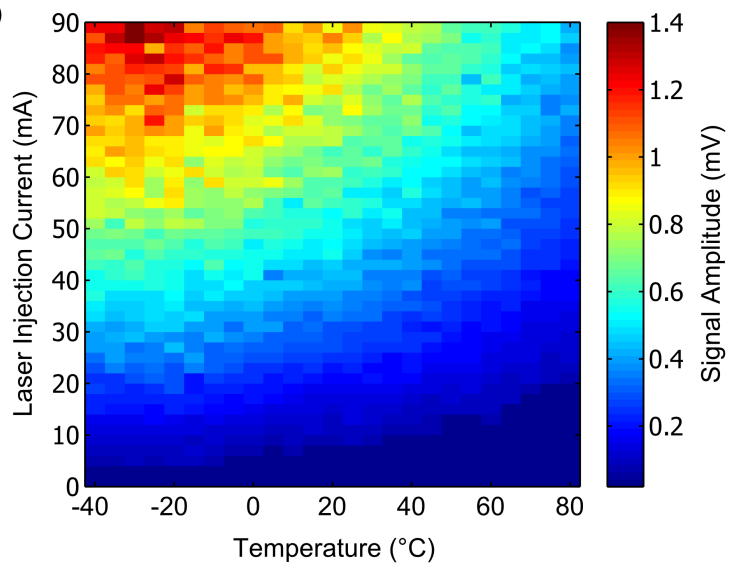

(b)

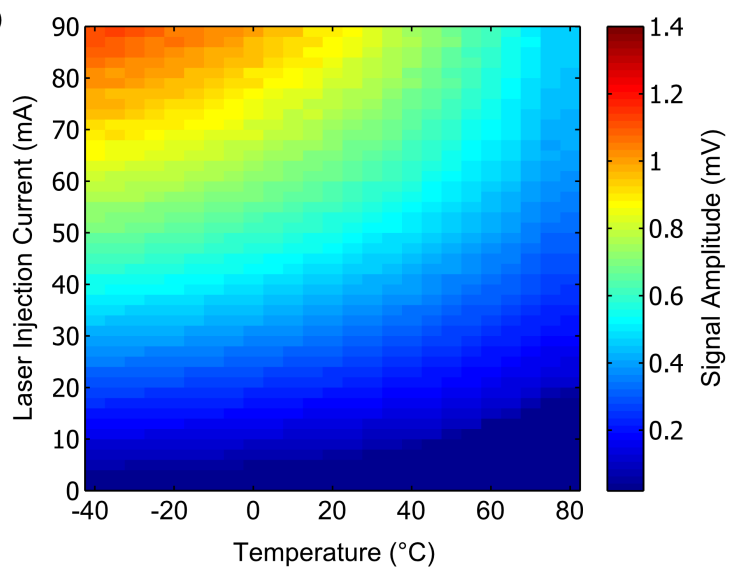

Fig. 7. PD signal strength as a function of the injection current and temperature: (a) Measured, and (b) modelled.

\section{Conclusion}

The simple model developed and presented here explicitly describes the dependence of both OFI PD and LV signals strengths under weak feedback on the system parameters, and in particular the laser injection current and operating temperature. Our model shows that the form of signal strength dependence on injection current is of entirely different character for PD and LV signals: PD signal strength increases with injection current, while LV signal strength is greatest just above the laser threshold and subsequently decreases with increasing injection current. This interesting phenomenon can be accounted for by including injection current dependence of slope efficiency. Moreover, the model offers a clear explanation of the experimentally observed monotonic decrease in the PD signal strength with temperature, as slope efficiency decreases with the temperature increase while threshold current increases. Meanwhile, the LV signal strength is proportional to temperature and slope efficiency, and the two opposing effects lead to less pronounced change of the signal strength over the full temperature range with a plateau at temperatures around $0{ }^{\circ} \mathrm{C}$. The model proposed here provides insight into the selection of injection current levels which maximise the 
(a)

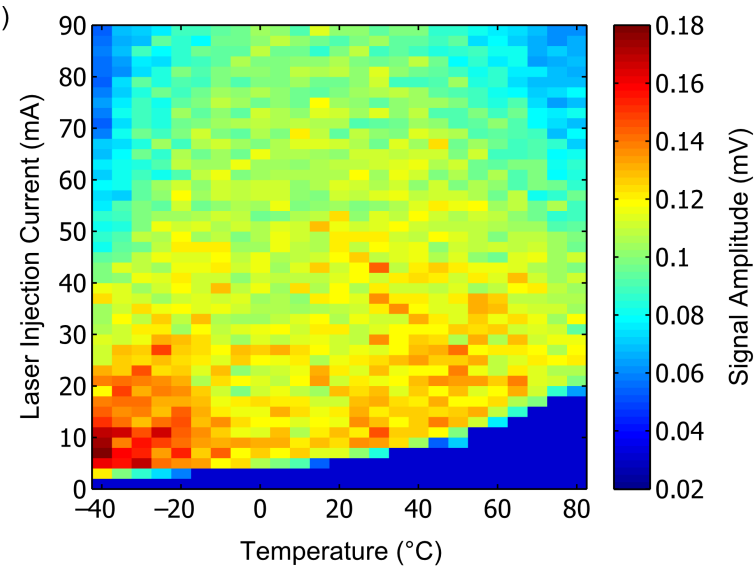

(b)

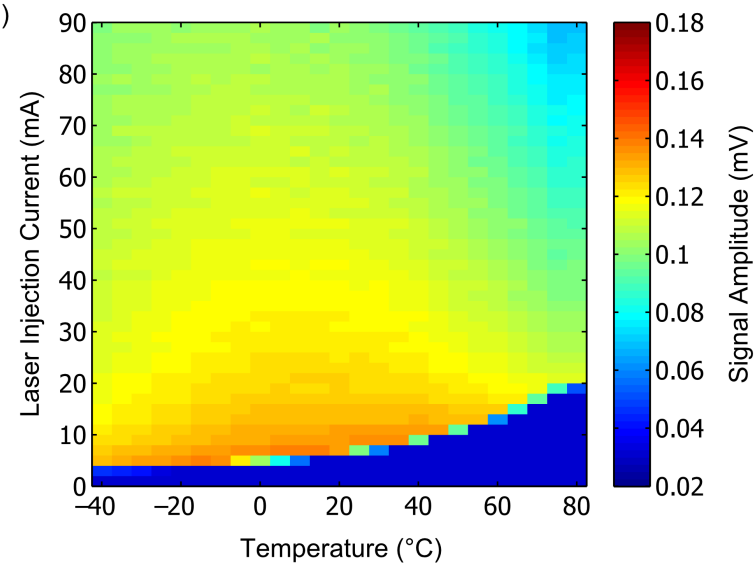

Fig. 8. LV signal strength as a function of the injection current and temperature: (a) Measured, and (b) modelled.

signal strength, and hence sensor sensitivity, for both OFI PD and LV schemes. The biasing strategy is radically different for these two schemes: for LV signals the optimal injection current is close to threshold, while PD schemes offer greater sensitivity at much higher bias currents. This simple model could be extended by further including the effects of noise, by taking into account the noise of the laser, the photodetector and the amplifier or by considering more complex lasing regimes with multiple transverse or longitudinal mode operation (as is usually the case for vertical-cavity surface-emitting lasers) starting from the generalised Lang and Kobayashi model instead of the standard model.

\section{Acknowledgments}

This research was supported under Campus France, the Conseil Régional de Midi-Pyrénées funding program, and Australian Research Council's Discovery Projects funding scheme (DP 120 103703). YLL acknowledges support under the Queensland Government's Smart Futures Fellowships programme.

\section{References}

[1] T. Bosch, C. Bes, L. Scalise, and G. Plantier, "Optical feedback interferometry," Encyclopedia of Sensors X, 120 (2006).

[2] R. Juskaitis, N. P. Rea, and T. Wilson, "Semiconductor laser confocal microscopy," Appl. Opt. 33, 578-584 (1994).

[3] J. R. Tucker, A. Mowla, J. Herbert, M. A. Fuentes, C. S. Freakley, K. Bertling, Y. L. Lim, R. S. Matharu, J. Perchoux, T. Taimre, S. J. Wilson, and A. D. Rakić, "Selfmixing sensing system based on uncooled vertical-cavity surface-emitting laser array: linking multichannel operation and enhanced performance," Opt. Lett. 39, 394-397 (2014).

[4] Y. Mitsuhashi, T. Morikawa, J. Shimada, K. Sakurai, and S. Mitsutsuka, "Polarization-rotated optical feedback in self-coupled optical pickup," Opt. Commun. 34, $309-310$ (1980).

[5] K. B. Rochford and A. H. Rose, "Simultaneous laserdiode emission and detection for fiber-optic sensor applications," Opt. Lett. 20, 2105-2107 (1995).

[6] R. S. Matharu, J. Perchoux, R. Kliese, Y. L. Lim, and A. D. Rakić, "Maintaining maximum signal-to-noise ratio in uncooled vertical-cavity surface-emitting laserbased self-mixing sensors," Opt. Lett. 36, 3690-3692 (2011).

[7] Y. Mitsuhashi, J. Shimada, and S. Mitsutsuka, "Voltage change across the self-coupled semiconductor laser." IEEE J. Quantum Electron. 17, 1216 - 1225 (1981).

[8] S. Donati, "Responsivity and noise of self-mixing photodetection schemes," IEEE J. Quantum Electron. 47, 1428-1433 (2011).

[9] M. Ravaro, S. Barbieri, G. Santarelli, V. Jagtap, C. Manquest, C. Sirtori, S. P. Khanna, and E. H. Linfield, "Measurement of the intrinsic linewidth of terahertz quantum cascade lasers using a near-infrared frequency comb," Opt. Express 20, 25654-25661 (2012).

[10] K. Bertling, Y. L. Lim, T. Taimre, D. Indjin, P. Dean, R. Weih, S. Höfling, M. Kamp, M. von Edlinger, J. Koeth, and A. D. Rakić, "Demonstration of the selfmixing effect in interband cascade lasers," Appl. Phys. Lett. 103, 231107 (2013).

[11] S. Donati, and R.-H. Horng, "The diagram of feedback regimes revisited," IEEE J. Sel. Top. Quantum Electron. 19, 1500309 (2013).

[12] K. Kou, X. Li, L. Li, and H. Xiang, "Injected current reshaping in distance measurement by laser self-mixing interferometry," Appl. Opt. 53, 6280-6286 (2014).

[13] A. A. Sahai, B. Kim, D. Choi, A. Locquet, and D. S. Citrin, "Mapping the nonlinear dynamics of a laser diode via its terminal voltage," Opt. Lett. 39, 56305633 (2014).

[14] R. Lang and K. Kobayashi, "External optical feedback effects on semiconductor injection laser properties," IEEE J. Quantum Electron. 16, 347 - 355 (1980).

[15] G. Acket, D. Lenstra, A. den Boef, and B. Verbeek, "The influence of feedback intensity on longitudinal mode properties and optical noise in index-guided semiconductor lasers," IEEE J. Quantum Electron. 20, 1163 1169 (1984).

[16] G. Giuliani and S. Donati, "Laser interferometry," in Unlocking Dynamical Diversity: Optical Feedback Effects on Semiconductor Lasers, D. M. Kane and K. A. Shore, 
eds. (John Wiley \& Sons, 2005), pp. 217-255.

[17] T. Taimre and A. D. Rakić, "On the nature of Acket's characteristic parameter $C$ in semiconductor lasers," Appl. Opt. 53, 1001-1006 (2014).

[18] J. Katz, S. Margalit, C. Harder, D. Wilt, and A. Yariv, "The intrinsic electrical equivalent-circuit of a laser diode," IEEE J. Quantum Electron. 17, 4-7 (1981).

[19] L. A. Coldren, S. W. Corzine, and M. L. Mashanovitch, Diode Lasers and Photonic Integrated Circuits (John Wiley \& Sons, 2012), 2nd ed.
[20] Y. L. Lim, P. Dean, M. Nikolić, R. Kliese, S. P. Khanna, M. Lachab, A. Valavanis, D. Indjin, Z. Ikonić, P. Harrison, E. H. Linfield, A. G. Davies, S. J. Wilson, and A. D. Rakić, "Demonstration of a self-mixing displacement sensor based on terahertz quantum cascade lasers," Appl. Phys. Lett. 99, 081108 (2011).

[21] T. Hertsens, "Measuring Diode Laser Characteristics," ILX Lightwave, Appl. Note 5, 3-5 (2005). 